\title{
Mathematical model of optimization of annealing regimes by the stress state for heat-sensitive glass elements of structures
}

\author{
Hachkevych O. ${ }^{1,2}$, Hachkevych M. ${ }^{1}$, Torskyy A. ${ }^{3}$, Dmytruk V.., 3 \\ ${ }^{1}$ Pidstryhach Institute for Applied Problems of Mechanics and Mathematics \\ National Academy of Sciences of Ukraine, 3-b Naukova Str., 79060, Lviv, Ukraine \\ ${ }^{2}$ Opole University of Tecnnology, 76 Prószkowska Str., Opole, 45-758, Poland \\ ${ }^{3}$ Centre of Mathematical Modelling IAPMM of Ukranian National Academy of Sciences, \\ 15 Dudayev Str., Lviv, 79005, Ukraine \\ ${ }^{4}$ Lviv Polytechnic National University, 12 S. Bandera Str., Lviv, 79013, Ukraine
}

(Received 15 January 2018)

\begin{abstract}
A mathematical model is constructed and a problem of optimization of annealing regimes of thermosensitive glass elements of constructions with the use of numerical methods and variational calculus is solved. As an example, the optimal regimes of annealing of specific glass plates for different values of permissible stresses are constructed and analyzed.
\end{abstract}

Keywords: thermal regimes in production processes, optimization of relatively stressed state, thermosensitive glass elements.

2000 MSC: 74A10, 74B10

UDC: 539.3

DOI: $10.23939 / \mathrm{mmc} 2018.02 .134$

\section{Introduction}

A glass element in which the residual temperature stresses being formed in the course of its manufacture (fusing, glass-ceramics joining, etc.) together with the operational stresses can lead to the destruction of the element, in order to reduce the level of these stresses by means of the relaxation is annealed. With this, the element is heated from the initial temperature to the given one (which should be lower than the temperature of the melting of the glass, as in the opposite case there may be more than deformations of the element). With the intention of lowering the level of the residual stresses by relaxation, the element is exposed to a given temperature for a certain time, and then is gradually cooled to a known temperature of the output from the furnace. The duration of the annealing regime depends on the level of the residual stresses that arose in the product during its manufacturing, as well as on the level of available temperature stresses for annealing.

When a glass product is annealed, the value of the permissible stresses is set for the heating zone from the initial temperature to the annealing temperature (maximal at the heating interval) and the cooling zone from this maximum to the temperature of the furnace output.

The paper deals with the problem of modeling and optimization by stresses of the annealing modes of glass elements of electric vacuum devices (EVD) in order to reduce the level of residual stresses in the way of their relaxation. As an element for calculations, the element of a small curvature, which is modeled by a plate free at the edges (butts) of the constant thickness $2 h$.

\section{Statement of the problem}

When constructing an optimization technique of annealing regimes, we assume that annealing is carried out by means of convective heating by the external environment from the side of one of the bases of the model element of the plate. In this case, at the bases $\gamma= \pm h$ of the plate, a variable in time 
temperature $t^{+}(\tau)$ (control function) is maintained, i.e.

$$
t(\gamma, \tau)=t^{+}(\tau) \quad \text { for } \quad \gamma= \pm h
$$

Here $t(\gamma, \tau)$ is the temperature calculated from the initial $t_{p}, \gamma$ is the thickness coordinate; $\tau$ is the time. Then the temperature in the plate satisfies the initial condition

$$
t(\gamma, 0)=t_{p}
$$

The distribution of temperature over the thickness of the plate, under its approximating by a cubic polynomial, in the case under consideration (for symmetric conditions of plate heating relatively to the median surface) will have the form [1]:

$$
t(\gamma, \tau)=\frac{a^{2} h^{2}}{2}\left(\frac{\gamma^{2}}{h^{2}}-\frac{1}{3}\right) \frac{d T_{1}}{d \tau}+T_{1}
$$

Here $T_{1}=\frac{1}{2 h} \int_{-h}^{h} t d \gamma$ is the temperature averaged over the thickness of the plate, which satisfies the following equation [1]

$$
a^{2} h^{2} \frac{d T_{1}}{d \tau}+3 T_{1}=3 t^{+}(\tau)
$$

obtained for the case of a plate from the corresponding relation (3) taking into account the boundary conditions (1) for $\gamma=h$. It should be mentioned that in literature the nonlinear analysis is often suggested to be applied to study plate and shell structures under thermal loading. As an illustrative example, in [2] the non-linear coupled partial differential equations derived in the von Karman sense and extended to thermal loading with the inclusion of curvature for a skew rectangular panel has been employed to analyze thermal stresses for movable edge boundary condition.

Taking into account the condition (2) and taking into account (3), we obtain:

$$
T_{1}(0)=t_{p}, \quad \frac{d T_{1}(0)}{d \tau}=0 .
$$

The temperature stresses in the thermosensitive plate free from the external load are described by the formulas given in [3], i.e.,

$$
\sigma_{1}=\sigma_{2} \equiv \sigma=\frac{E}{1-\nu}\left(e_{t}-\Phi\right),
$$

where $E$ is the modulus of elasticity; $\Phi(t)$ is the total pure thermal deformation determined by the formula $\Phi(t)=\int_{t_{0}}^{t} \alpha_{t}(\xi) d \xi ; \alpha_{t}$ is the coefficient of linear temperature expansion; $e_{t}=\frac{1}{2 h} \int_{-h}^{h} \Phi(t) d \gamma$; $\nu$ is the Poisson's ratio.

With this, the components of the elastic deformation of the median surface will be

$$
\varepsilon_{1}=\varepsilon_{2}=e_{t}, \quad \chi_{1}=\chi_{2}=0,
$$

where $\varepsilon_{1}, \varepsilon_{2}$ are the components of tensile strain (compression); $\chi_{1}, \chi_{2}$ are the deformation changes of curvature.

In view of the above, the initial problem of optimization by the stress state of the annealing regimes of the considered glass element is reduced to the construction of the optimal by this state the annealing regimes the plate free at the edges, on the surfaces $\gamma= \pm h$ of which the temperature $t^{+}(\tau)$ is known at given constraints on its change and on the corresponding temperature stresses $\sigma^{+}$for $\gamma= \pm h$.

As it is known [4-8], the strength of glass on the surface of the plate is $3-4$ times less than the its strength inside. The compression resistance is $8-10$ times higher than tensile strength. Therefore, in determining the optimal by stresses annealing regimes for thin glass plates, we will take into account the limitations on the magnitude of surface temperature stresses. 
The plate must be heated in a convective manner from the initial temperature $t_{\mathrm{p}}$ to the maximal $t_{0}$ for the time $\tau_{0}$ on the surface $\gamma=h$, then this temperature must be kept for the time $\tau_{B}$, and then the surface must be cooled down to the final temperature $t_{*}\left(t_{*} \leqslant t_{0}\right)$ for the time $t_{*}$ under certain constraints on the parameters of thermostressed state and the ratio of heating. The control function is the temperature $t^{+}(\tau)=t(h, \tau)$ of the outer surface of the plate, which is an unknown function and satisfies the following conditions (in accordance with the stated purpose of heating):

$$
\begin{gathered}
t^{+}(0)=t_{\mathrm{p}} ; \quad t^{+}(\tau)=t_{0} \quad \text { for } \quad \tau_{0} \leqslant \tau \leqslant \tau_{01} ; \quad t^{+}\left(\tau_{*}\right)=t_{*} ; \\
V_{T 1} \leqslant \frac{d t^{+}(\tau)}{d \tau} \leqslant V_{T 2} ; \quad\left(\frac{d t^{+}(\tau)}{d \tau}\right)_{\tau=\tau_{0}}=0 ; \quad\left(\frac{d t^{+}(\tau)}{d \tau}\right)_{\tau=\tau_{01}}=0,
\end{gathered}
$$

reflecting the purpose of heating and the specifics of the heat treatment of glass products [9], as well as the smoothness of the control function at certain moments of time (the conditions allowing the practical realization of optimal regimes). In (8), $V_{T 1}, V_{T 2}$ are the given permissible heating ratios.

To ensure the strength of the considered plate over the entire heating interval $\left[0, \tau_{*}\right]$, we assume that the parameters of the thermostressed state change within the given boundaries

$$
\sigma_{0}^{ \pm} \leqslant \sigma^{ \pm} \leqslant \sigma_{*}^{ \pm}
$$

where $\sigma_{0}^{ \pm} \leqslant 0, \sigma_{*}^{ \pm} \geqslant 0, \sigma_{0}^{ \pm}, \sigma_{*}^{ \pm}$are given values of permissible stresses. From here, the signs "+", "-" are used to denote the functions and values on the outer and inner surfaces of the plate, respectively.

In the formulation of optimization problems for a stress-strain state, it is important to choose the optimality criterion (which must be an energy or other measure of the quantities by which processes are optimized). For homogeneous shells, as a rule, they choose the appropriate functional, which in an integral sense reflects the goal of optimization. In particular, in the constructing the optimal modes of technological heating of the shells there is used a functional of elastic deformation energy [1], which is the measure of the stress state in the whole domain of the shell for an arbitrary time $\tau$ and it is related to the thermal strength of the glass product at the stages of heating-cooling. In the plates, the resulting temperature stresses in the process of heat treatment essentially depend on the nature of the temperature distribution. At the same time, the maximal normal stresses (because of which, according to the first theory of strength, the glass crackes) arise on the surface of the plate. Therefore, when optimizing the stress state, it is expedient to use a local optimality criterion, which is a more precise measure of a stress state. As such a criterion of optimality, we take the functional of maximal normal stresses

$$
N=\max \left\{\sigma\left(\alpha_{1}, \alpha_{2}, \gamma, \tau\right)\right\}, \quad \alpha_{1}, \alpha_{2}, \gamma \in\left(V_{0}\right) ; \quad 0 \leqslant \tau \leqslant \tau_{*},
$$

where $\tau_{*}$ is the duration of the heat treatment, $V_{0}$ is the domain of change of the curvilinear coordinates in the three-dimensional Euclidean space occupied by the plate.

The problem is formulated for searching a control function $f$ which provides a minimum of the functional (10) on the set of functions describing the thermostress state of the plate and satisfying the system of the corresponding correlations of the thermomechanics and the conditions for fixing the surfaces of the plate.

We will construct a solution of the formulated problem based on the principle of stepwise parametric optimization, and for the realization of the search phase of the conditional minimum of the functional (10) we apply the method of local variations [10], which is based on the variations in the space of states of the control function under a known solution of the direct problem (which describes the thermostress state of the plate at the given thermal and mechanical boundary conditions for the known values of the control function).

The method of local variations is based on the known values of varying values, which are the solution of direct problems. Therefore, when they practically use this method for calculating the values of values, which should be compared, it is necessary to have the initial (input) value of the control function at the whole interval of the argument change for the most possible accounting of the 
technological, physical and geometric features of it, for which it is possible to construct solutions of the direct problems. In this connection, it is relevant to construct such an initial approximation of the control function (using analytical methods) which would take into account as many as possible of the mentioned features.

\section{Solving the direct problem}

For the given law of heating (at the known temperature $t^{+}(\tau)$ of the surface), Eq. (4) is solved by the method of least squares for finite-element approximation of the heating mode according to the method described in $[11,12]$. We introduce the operator notations and present (4) in the form

$$
A u=f,
$$

where $u=\left(T_{1}\right), f=\left(t^{+}\right), A=\left(\frac{a^{2} h^{2}}{3} \frac{d}{d \tau}+1\right)$.

Let us divide the heating interval $\left[0, \tau_{*}\right]$ into the $p-1$ finite element in time with nodes $1,2,3, \ldots, p$. The unknown functions $T_{1}$ we present on the interval $\left[0, \tau_{*}\right]$ by the functions of the shape and values of these functions in the nodes. Then, at $i$-th step we will have the following:

$$
T_{1}(\tau)=G_{1}(\tau) T_{1}^{i}+G_{2}(\tau) T_{1}^{i+1}
$$

or in the matrix form

$$
T_{1}=\left[G_{1} G_{2}\right]\left[\begin{array}{c}
T_{1}^{i} \\
T_{1}^{i+1}
\end{array}\right]
$$

Here $\tau_{i} \leqslant \tau \leqslant \tau_{i+1}, T_{1}^{i}, T_{1}^{i+1}$ are the values of the function $T_{1}$ at the points $\tau_{i}, \tau_{i+1}$, and $G_{1}(\tau), G_{2}(\tau)$ are the functions of the shape.

For the function of the shape, we choose a polynomial of the first degree satisfying the following conditions:

$$
G_{1}\left(\tau_{i}\right)=1, \quad G_{1}\left(\tau_{i+1}\right)=0, \quad G_{2}\left(\tau_{i}\right)=0, \quad G_{2}\left(\tau_{i+1}\right)=1 .
$$

With this:

$$
G_{1}(\tau)=\frac{\tau_{i+1}}{\tau_{i+1}-\tau_{i}}-\frac{\tau}{\tau_{i+1}-\tau_{i}}, \quad G_{2}(\tau)=-\frac{\tau_{i}}{\tau_{i+1}-\tau_{i}}+\frac{\tau}{\tau_{i+1}-\tau_{i}} .
$$

Let the vector $q^{(i)}$ a set of values of the desired functions in the nodes $i$-th element, and $\tilde{G}$ is a matrix, whose elements are the functions of the shape. Then

$$
u^{(i)}=\left[G_{1}, G_{2}\right]\left[\begin{array}{c}
T_{1}^{i} \\
T_{1}^{i+1}
\end{array}\right]=\tilde{G} q^{(i)}, \quad f^{(i)}=\tilde{G} \delta, \quad \text { where } \quad \delta=\left(\begin{array}{c}
t_{i}^{+} \\
t_{i+1}^{+}
\end{array}\right) .
$$

Minimizing the function of the least squares method

$$
\tilde{I}^{(i)}=\int_{\tau_{j}}^{\tau_{j+1}}\left(q^{(i) \mathrm{T}} \tilde{N}^{\mathrm{T}} A^{\mathrm{T}} A \tilde{N} q^{(i)}-2 q^{(i) T} \tilde{N}^{\mathrm{T}} A^{\mathrm{T}} \tilde{N} \delta\right) d \tau
$$

For the $i$-th element for all parameters $q^{(i)}$ we obtain a system of linear algebraic equations with respect to unknown values of the function $T_{1}$ in the nodes of the $i$-th element. Summing by all elements we obtain a system of linear algebraic equations

$$
[K]\{\delta\}=\{S\}
$$

with respect to the unknown values of the function $T_{1}$ in the nodes of splitting the interval $0 \leqslant \tau \leqslant \tau_{*}$ with the points $\tau_{i}=i \frac{\tau_{*}}{p}$ into $p$ equal parts. Here:

$$
[K]=\sum_{i} K^{i}, \quad\{S\}=\sum_{i} S^{i}, \quad\{\delta\}=\sum_{i} q^{(i)}, \quad q^{(i)}=\left(T_{1}^{i}, T_{1}^{i+1}\right)^{\mathrm{T}},
$$

the transpose matrix is indicated by the symbol " $T$ ". 
The expressions for the elements of the matrix $K^{i}, S^{i}$ (for the $i$-th element) will be written in expanded form:

$$
\begin{gathered}
K_{11}=\frac{a^{4} h^{4}}{9} \frac{1}{p}-\frac{a^{2} h^{2}}{3}+\frac{p}{3}, \quad K_{12}=-\frac{a^{4} h^{4}}{9} \frac{1}{p}+\frac{p}{6}, \quad K_{21}=K_{12}, \\
K_{22}=\frac{a^{4} h^{4}}{9} \frac{1}{p}+\frac{a^{2} h^{2}}{3}+\frac{p}{3}, \quad S_{1}=\left(-\frac{a^{2} h^{2}}{6}+\frac{p}{3}\right) t_{i}^{+}+\left(-\frac{a^{2} h^{2}}{6}+\frac{p}{6}\right) t_{i+1}^{+}, \\
S_{2}=\left(\frac{a^{2} h^{2}}{6}+\frac{p}{6}\right) t_{i}^{+}+\left(\frac{a^{2} h^{2}}{6}+\frac{p}{3}\right) t_{i+1}^{+},
\end{gathered}
$$

where $p=\tau_{i+1}-\tau_{i}$.

We also note that to achieve the same accuracy of the solution by the method of finite differences, it is necessary to considerably thicken the division with respect to time (which leads to an increase in the duration of calculations).

To solve the system (17), we use the Gauss method in Kholeskii modification [11]. For the known values of averaged temperature $T_{1}^{(i)}$, using the correlation (3), we calculate the temperature for the time moment $\tau_{i}$ and for arbitrary values of the thickness coordinate $\gamma$. With this, we use the appropriate numerical differentiation formulas to calculate the derivatives $T_{1}$ in time [13-15].

To calculate purely thermal deformation $\Phi^{ \pm}$and its averaged characteristic $e_{t}$, we use the method described in [12]. At the same time, the dependence of the coefficient of linear thermal expansion on the temperature will be given by means of piecewise-linear functions in the form

$$
\alpha_{t}(t)=\sum_{p=1}^{q}\left(b^{(p)} t+d^{(p)}\right) S_{+}\left(t_{(p)}-t\right),
$$

where $t_{(p-1)} \leqslant t \leqslant t_{(p)}, S_{+}$is the function of jump, $b^{(p)}, d^{(p)}$ are the parameters describing piecewiselinear dependence.

In this case, the distribution (3) of the temperature in the shell thickness is given in the form

$$
t(\gamma, \tau)=A(\tau) \gamma^{3}+B(\tau) \gamma^{2}+C(\tau) \gamma+D(\tau),
$$

where

$$
A=0, \quad B=\frac{a^{2}}{2} \frac{d T_{1}}{d \tau}, \quad C=0, \quad D=T_{1}-\frac{a^{2} h^{2}}{6} \frac{d T_{1}}{d \tau}
$$

For the known values $\Phi^{ \pm}, e_{t}$, we find the values of the stresses on the surface of the plate by the formula (6).

The algorithm for constructing a solution to an extreme problem consists of two iterative processes: the variation of the value of the control function $t^{+} \equiv\left\{t_{(n)}^{+}\left(\tau_{i}\right)\right\}$ at discrete moments at a fixed step $\delta$ and the splitting of this step.

In approximating, the desired control function $t_{(n-1)}^{+}\left(\tau_{i}\right)$ is chosen so that the thermal conditions (8) and the constraints (9) are fulfilled. In this case, it is necessary to have a solution of the direct problem, i.e., the values of temperature and stresses at the given conditions of convective heat transfer. The fulfillment of the constraints (9) is provided by the above-described algorithm by comparing the stress components determined by the numerical-analytic method from the direct problem with the given permissible values. To find the following approximation of the control function $t_{(n)}\left(\tau_{i}\right)$, for the three values $t_{(n-1)}^{+}\left(\tau_{i}\right) \pm \delta_{(n-1)} ; t_{(n-1)}^{+}\left(\tau_{i}\right)$ of this function; (obtained in the previous approximation) we calculate the value of the optimality criterion (10) using the solution of the direct problem. The step $\delta_{(n-1)}$ of variation (sufficiently small positive number, constant for the specific $n$ ) is taken for $n=2$ to be equal to the maximal gradient of the control function in the initial approximation. The desired function $t_{(n)}^{+}\left(\tau_{i}\right)$ is the one for which the value of the criterion (10) is minimal and the conditions (8), (9) are satisfied. 
The following approximations of the control function are obtained using the previous algorithm with the step splitting by the algorithm $\delta_{(n)}=\delta_{(n-1)} / 2, n=2,3,4, \ldots$. The iteration process continues to fulfill the following condition

$$
\left\{t_{(n+1)}^{+}\left(\tau_{i}\right)\right\} \equiv\left\{t_{(n)}^{+}\left(\tau_{i}\right)\right\}, \quad \delta_{(n)} \leqslant \varepsilon,
$$

where $\varepsilon$ is a given small number, $\tau_{i}=i \frac{\tau_{*}}{N}(i=\overline{1, N-1})$.

The search for the conditional minimum of the functional (10) is carried out by comparing the stresses in the domain of change in the thickness coordinate $\gamma$ with the step $\Delta \gamma(\Delta \gamma=0.1 \mathrm{~h})$, which we calculate for the known values of the temperature (found in the previous step) at the same nodes,

For the proposed iteration optimization algorithm, it is important to choose the initial approximation of the values of the control function, which determines the convergence of the iterative process. The initial values of the control function are determined on the basis of the solution of the optimization problem by the stress state of the annealing modes of a free at the edges homogeneous plate on the surfaces $\gamma= \pm h$ of which the temperature $t^{+}(\tau)$ is given with certain constraints on its change and the corresponding temperature stresses $\sigma^{+}$for $\gamma= \pm h$, and also with a constant coefficient of temperature expansion.

Given the symmetry of the problem and the technological conditions, we record the following constrants on the temperature and temperature stresses on the surface $\gamma=+h$ :

- the function $t^{+}(\tau)$ for $0 \leqslant \tau<\tau_{1}$ is positive and limited by the values of $t_{0}$. For $\tau=0$ $t^{+}(\tau)=t_{p}$, and for $\tau \geqslant \tau_{1}-t^{+}(\tau)=t_{1}$, i.e.:

$$
t_{p} \leqslant t^{+}(\tau) \leqslant t_{0}, \quad 0 \leqslant \tau<\tau_{1} ; \quad t^{+}(\tau)=t_{1}, \quad \tau \geqslant \tau_{1},
$$

where $\tau_{1}$ is the time of treatment end;

- the function $t^{+}(\tau)$ subordinates to the system of functional conditions of the form

$$
\int_{0}^{\tau_{1}} \tau^{i} t^{+}(\tau) d \tau=A_{i}, \quad(i=\overline{0, n})
$$

where $A_{i}$ are the arbitrary constants (which can be used to ensure the smoothness of the desired modes and the implementation of certain physical constraints, in particular energy one);

- the temperature stresses $\left.\sigma^{+}(h, \tau) \equiv \sigma(\gamma, \tau)\right|_{\gamma=+h}$ on the surface $\gamma=+h$ change in the given limits, i.e.:

$$
\sigma_{0}^{+} \leqslant \sigma^{+} \leqslant \sigma_{*}^{+}, \quad \sigma_{*}^{+} \geqslant 0, \quad \sigma_{0}^{+} \leqslant 0 .
$$

The formulated optimization problem is solved using the methods of the variational calculus on the basis of minimization of the functional of the plate elastic deformation energy during the heating [1], which in the case under consideration has the form

$$
M=\frac{2 a^{4} h^{5} E \alpha_{t}^{2}}{45(1-\nu)} \int_{0}^{\tau_{1}}\left(\frac{d T_{1}}{d \tau}\right)^{2} d \tau
$$

and it is a functional given on the set of functions $T_{1}$.

Using the approach [1], the constraints (22), (23), (24) on temperature $t^{+}(\tau)$ and temperature stresses $\sigma^{+}$, taking into account the expressions (3), (4), (6) and functions with a limited change domain [1] can be represented in the form:

$$
\begin{gathered}
\frac{a^{2} h^{2}}{3} \frac{d T_{1}}{d \tau}+T_{1}-\frac{t_{0}}{2}\left(1+\sin \phi_{1}^{+}(\tau)\right)=0, \quad \int_{0}^{\tau_{1}} \tau^{i}\left(1+\sin \phi_{1}^{+}(\tau)\right) d \tau=A_{i}, \quad i=\overline{0, n}, \\
\frac{a^{2} h^{2}}{3} \frac{d T_{1}}{d \tau}+\frac{(1-\nu)\left(\sigma_{*}^{+}-\sigma_{0}^{+}\right)}{2 E \alpha_{t}}\left(\sin \phi_{11}^{+}(\tau)+\frac{\sigma_{*}^{+}+\sigma_{0}^{+}}{\sigma_{*}^{+}-\sigma_{0}^{+}}\right)=0
\end{gathered}
$$


where $\phi_{1}^{+}$and $\phi_{11}^{+}$are arbitrary functions. Then finding the solution of the formulated optimization problem is reduced to the determination of the extremals of the functional (25) on the set of functions $T_{1}, \phi_{1}^{+}, \phi_{11}^{+}$satisfying the conditions (26). The solving of such a problem about a conditional extremum is reduced to finding the unconditional extremum of the following functional

$$
\begin{array}{r}
M^{*}=\frac{2 h E \alpha_{t}^{2}}{3(1-\nu)} \int_{0}^{\tau_{1}}\left\{\frac{a^{4} h^{4}}{15}\left(\frac{d T_{1}}{d \tau}\right)^{2}+\lambda_{1}(\tau)\left[\frac{a^{2} h^{2}}{3} \frac{d T_{1}}{d \tau}+\frac{\left(\sigma_{*}^{+}-\sigma_{0}^{+}\right)(1-\nu)}{2 E \alpha_{t}}\left(\sin \phi_{11}^{+}(\tau)+\frac{\sigma_{*}^{+}+\sigma_{0}^{+}}{\sigma_{*}^{+}-\sigma_{0}^{+}}\right)\right]\right. \\
\left.+\lambda_{2}(\tau)\left[\frac{a^{2} h^{2}}{3} \frac{d T_{1}}{d \tau}+T_{1}-\frac{t_{0}}{2}\left(1+\sin \phi_{1}^{+}(\tau)\right)\right]+\frac{t_{0}}{2} \sum_{i=0}^{n} \lambda_{i 0} \tau^{i}\left(1+\sin \phi_{1}^{+}(\tau)\right)\right\} d \tau, \quad(27)
\end{array}
$$

where $\lambda_{1}, \lambda_{2}, \lambda_{i 0}, i=\overline{0, n}$ are the corresponding Lagrange multipliers. From the necessary condition for the extremum of the functional (27), we obtain the following system of Euler equations:

$$
\begin{aligned}
\frac{d^{2} T_{1}}{d \tau^{2}}+\frac{5}{2 a^{2} h^{2}}\left(\frac{d \lambda_{1}}{d \tau}+\frac{d \lambda_{2}}{d \tau}\right)- & \frac{15}{2 a^{4} h^{4}} \lambda_{2}=0, \quad \lambda_{1} \cos \phi_{11}^{+}(\tau)=0 \\
& \left(\lambda_{2}-\sum_{i=0}^{n} \lambda_{i 0} \tau^{i}\right) \cos \phi_{1}^{+}(\tau)=0
\end{aligned}
$$

From this system of equations and the conditions (26), we find the desired functions $T_{1}, \phi_{1}^{+}, \phi_{11}^{+}$, and the Lagrange multipliers $\lambda_{1}(\tau), \lambda_{2}(\tau)$ and $\lambda_{i 0}$. By means of the already determined (known) mentioned quantities, we consistently find the temperature $t$ in the plate and the temperature $t^{+}$on its surface, and then by the formula

$$
\sigma_{1}=\sigma_{2} \equiv \sigma=\frac{E \alpha_{t}}{1-\nu}\left(T_{1}-t\right)
$$

we find the stresses in the plate.

We note that the Lagrange multipliers $\lambda_{i 0}$ included in the Euler equations system (28) are used to satisfy the given constraints at the temperature and stress levels at discrete moments of time [1].

\section{The regime under constraints on tensile stresses}

Let us consider the case when the constraints are given only on the tensile stresses $\sigma^{+}$on the surface $\gamma=+h$ of the plate, and the temperature $t^{+}$on this surface at the initial moment of time $\tau=0$ is equal to $t_{p}$. For $\tau \geqslant \tau_{1}\left(\tau_{1}\right.$ is the completion time of the annealing process $) t^{+}=t_{1}$ and for $\tau=\tau_{0}$ $\left(0<\tau_{0}<\tau_{1}\right), t^{+}$reaches the maximal value $t_{0}$, i.e.

$$
t^{+}(0)=t_{p}, \quad t^{+}\left(\tau_{1}\right)=t_{1}, \quad t^{+}\left(\tau_{0}\right)=t_{0}, \quad \dot{t}\left(\tau_{0}\right)=0,
$$

where the point above the letter means the derivative by time.

At the time interval $\tau_{0} \div \tau_{01}$ (the time of exposure), the temperature $t^{+}$on the plate surface $\gamma=+h$ is constant and equal to $t_{0}$, i.e.

$$
t^{+}(\tau)=t_{0} \quad \text { for } \quad \tau_{0} \leqslant \tau \leqslant \tau_{01}
$$

Tensile thermal stresses $\sigma^{+}$on this surface under such a thermal regime do not exceed the permissible ones, i.e.

$$
\sigma^{+} \leqslant \sigma_{*}^{+}, \quad \text { where } \sigma_{*}^{+} \geqslant 0 .
$$

The values of permissible stresses $\sigma_{*}^{+}$at all stages of the annealing process are different and are set as follows. 
At the stage of heating the element from the initial temperature $t_{p}$ to the temperature of exposure $t_{0}$, i.e., for $0 \leqslant \tau \leqslant \tau_{0}$, the permissible level of stress $\sigma_{*}^{+}$is determined by the formula:

$$
\sigma_{*}^{+}=[\sigma]-\sigma_{k},
$$

where $[\sigma]$ is the allowable value of tensile stresses for glass [5]; $\sigma_{k}$ are the residual stresses in the element, which were formed during its manufacture (before the annealing process).

At the stage of exposure at a given temperature and cooling to the temperature of the lower annealing limit of a glass product, we have:

$$
\sigma_{*}^{+}=[\sigma]-\sigma_{k}^{*}(\tau) .
$$

Here $\sigma_{k}^{*}(\tau)$ are the residual stresses, the magnitudes of which decrease due to the relaxation from the value $\sigma_{k}$ to $\sigma_{k}^{*}\left(\tau_{01}\right)[5]$.

For the exposure at the given (maximal) temperature of the annealing mode, the temperature is equalized in the calculated glass element and as a consequence we have the decrease of the temperature gradients that occur in it. During the exposure at maximal annealing temperature, the viscosity of the glass decreases and the relaxation of residual stresses occurs. In the literature $[4,5]$, it is noted that the maximal relaxation of stresses occurs when the viscosity of glass is $10^{13} \mathrm{~Pa} \cdot \mathrm{s}$ (corresponding to a certain temperature of annealing) and for some (the duration of which is established experimentally for the glass types under consideration and the characteristic sizes of the product) time of exposure for this viscosity. Accordingly, for a particular brand of glass there is a known annealing temperature and the duration of exposure for it. For example, for a plate of C92 glass with a thickness $2 h=14 \mathrm{~mm}$, it is established that the maximal annealing temperature is $480-520^{\circ} \mathrm{C}$, and the duration of exposure at this temperature is about 20 minutes [3]) (such thickness has an onboard part of the screens of specific electric-vacuum devices (EVD)).

At the cooling stage (from the temperature of the lower annealing limit and to the given temperature of the output from the annealing furnace) the permissible stresses will be:

$$
\sigma_{*}^{+}=[\sigma]-\sigma_{k}^{*}\left(\tau_{01}\right) .
$$

When constructing optimal thermal regimes, as an initial we use the solution of an extreme problem without taking into account the constraints on the temperature $t^{+}$and temperature stresses $\sigma^{+}$. In this case, in the system of Euler equations (28), it is necessary to take $\lambda_{1}=0, \lambda_{2}=\sum_{i=0}^{n} \lambda_{i 0} \tau^{i}$. Then this system of equations is reduced to one equation from which we define the function $T_{1}$. With this

$$
\ddot{T}_{1}+\frac{5}{2 a^{2} h^{2}} \sum_{i=0}^{n} \lambda_{i 0} \tau^{i-1}\left(i-\frac{3 \tau}{a^{2} h^{2}}\right)=0 .
$$

The solutions of Eq. (36) are constructed at each of the time intervals $0 \leqslant \tau \leqslant \tau_{0}, \tau_{0} \leqslant \tau \leqslant \tau_{01}$ and $\tau_{01} \leqslant \tau \leqslant \tau_{1}$ by satisfying the conditions (30), (31) for the temperature $t^{+}$with the help of the representation (3) and verification the restrictions (32) on the temperature stresses $\sigma^{+}$. If at any of the mentioned stage the temperature regime $t^{+}$constructed on the basis of the solution of an extreme problem, causes in the plate at some moment $\tau_{02}$ the temperature stresses $\sigma^{+}$which exceed the permissible temperatures $\sigma_{*}^{+}$allowable for $t^{+}\left(\tau_{02}\right)<t_{0}$, then from this moment of time (using expressions (29) and the constraints (26) on the temperature stresses $\sigma^{+}$, as well as the system of Euler equations (28)), we proceed to the restriction regime $\sigma^{+}=\sigma_{*}^{+}$. Having determined the temperature regime $t^{+}$using this principle at all stages of heat treatment and ensuring the condition of its continuity in time, we obtain an optimal annealing by stresses regime for this element. 
Let us construct the solutions of Eq. (36) at each of the above-mentioned stages. When $0 \leqslant \tau \leqslant \tau_{0}$, the solution of this equation at $n=2$ is

$$
T_{1}(\tau)=\frac{5}{2}\left[\frac{3}{2} \lambda_{00} \tau^{2}+\lambda_{10} \frac{\tau^{2}}{2}(\tau-1)+\lambda_{20} \tau^{3}\left(\frac{\tau}{4}-\frac{1}{3}\right)\right]+C_{1} \tau+C_{2}
$$

where $\lambda_{00}, \lambda_{10}, \lambda_{20}, C_{1}, C_{2}$ are some constants determined from the proper of the above-mentioned conditions:

$$
t^{+}(0)=t_{p}, \quad t^{+}\left(\tau_{0}\right)=t_{0}, \quad \frac{d t^{+}\left(\tau_{0}\right)}{d \tau}=0,
$$

which for the function $T_{1}$ have the form:

$$
T_{1}(0)=t_{p}, \quad \dot{T}_{1}(0)=0, \quad T_{1}\left(\tau_{0}\right)=t_{0}, \quad \dot{T}_{1}\left(\tau_{0}\right)=\ddot{T}_{1}\left(\tau_{0}\right)=0 .
$$

From these initial conditions we obtain: $C_{1}=0, C_{2}=t_{p}$. Introduce the notations:

$$
\mu_{1}=\frac{\lambda_{00}}{t_{0}}, \quad \mu_{2}=\frac{\lambda_{10}}{t_{0}}, \quad \mu_{3}=\frac{\lambda_{20}}{t_{0}} .
$$

Then the expressions for $T_{1}(\tau), t^{+}(\tau), \sigma^{+}(\tau)$ at the heating stage $0 \leqslant \tau \leqslant \tau_{0}$ will be recorded:

$$
\begin{aligned}
T_{1}(\tau) & =\frac{5}{2} t_{0}\left[\mu_{1} \frac{3}{2} \tau^{2}+\mu_{2} \frac{\tau^{2}}{2}(\tau-1)+\mu_{3} \tau^{3}\left(\frac{\tau}{4}-\frac{1}{3}\right)\right]+t_{p} \\
t^{+}(\tau) & =\frac{5}{2} t_{0}\left[\mu_{1} \tau\left(1+\frac{3}{2} \tau\right)+\mu_{2} \tau\left(\frac{\tau^{2}}{2}-\frac{1}{3}\right)+\mu_{3} \tau^{2}\left(\frac{\tau^{2}}{4}-\frac{1}{3}\right)\right]+t_{p} \\
\sigma^{+}(\tau) & =-\frac{E \alpha_{t} t_{0}}{3(1-\nu)} \frac{5}{2}\left[3 \mu_{1} \tau+\mu_{2} \tau\left(\frac{3}{2} \tau-1\right)+\mu_{3} \tau^{2}(\tau-1)\right]
\end{aligned}
$$

Since, for $\tau_{0} \leqslant \tau \leqslant \tau_{01}$ we have $t^{+}(\tau)=t_{0}$, then from the equation

$$
\frac{a^{2} h^{2}}{3} \frac{d T_{1}}{d \tau}+T_{1}=t_{0}
$$

which follows from (4), taking into account the structure of its solution, we find

$$
T_{1}(\tau)=C_{1} \exp \left(-\frac{3 \tau}{a^{2} h^{2}}\right)+t_{0}
$$

From the conditions of continuity of the temperature field for $\tau=\tau_{0}$, i.e.:

$$
T_{1}\left(\tau_{0}-0\right)=T_{1}\left(\tau_{0}+0\right), \quad \dot{T}_{1}\left(\tau_{0}-0\right)=\dot{T}_{1}\left(\tau_{0}+0\right),
$$

we determine $C_{1}$. With this $C_{1}=0$. Therefore, for $\tau_{0} \leqslant \tau \leqslant \tau_{01}$ we have:

$$
T_{1}(\tau)=t_{0}, \quad t^{+}(\tau)=t_{0}, \quad \sigma^{+}(\tau)=0 .
$$

The stage $\tau_{01} \leqslant \tau \leqslant \tau_{1}$ is described by Eq. (36). The solution of this equation taking into account the conjugation conditions for the temperature $t(\gamma, \tau)$ at $\tau=\tau_{01}$ under and the thermal conditions at $\tau=\tau_{1}$, will be

$$
T_{1}(\tau)=t_{0}\left[\nu_{1} \frac{3}{2} \tau^{2}+\nu_{2} \frac{\tau^{2}}{2}(\tau-1)+\nu_{3} \tau^{3}\left(\frac{\tau}{4}-\frac{1}{3}\right)+\nu_{4} \tau+\nu_{5}\right] .
$$


Constants $\nu_{1} \div \nu_{5}$ are determined from the following conditions:

$$
\begin{array}{lll}
T_{1}\left(\tau_{01}-0\right)=T_{1}\left(\tau_{01}+0\right), & \dot{T}_{1}\left(\tau_{01}-0\right)=\dot{T}_{1}\left(\tau_{01}+0\right), \\
\ddot{T}_{1}\left(\tau_{01}-0\right)=\ddot{T}_{1}\left(\tau_{01}+0\right), & T_{1}\left(\tau_{1}\right)=t_{1}, & \dot{T}_{1}\left(\tau_{1}\right)=0 .
\end{array}
$$

By taking into account (3), (29) and (46), we obtain the following expressions for the functions $t^{+}(\tau)$ and $\sigma^{+}(\tau)$ :

$$
\begin{aligned}
t^{+}(\tau) & =t_{0}\left[\nu_{1} \tau\left(1+\frac{3}{2} \tau\right)+\nu_{2} \tau\left(\frac{\tau^{2}}{2}-\frac{1}{3}\right)+\nu_{3} \tau^{2}\left(\frac{\tau^{2}}{4}-\frac{1}{3}\right)+\nu_{4}\left(\tau+\frac{1}{3}\right)+\nu_{5}\right] \\
\sigma^{+}(\tau) & =-\frac{E \alpha_{t} t_{0}}{3(1-\nu)}\left[3 \nu_{1} \tau+\nu_{2} \tau\left(\frac{3}{2} \tau-1\right)+\nu_{3} \tau^{2}(\tau-1)+\nu_{4}\right]
\end{aligned}
$$

Now consider the cooling stage $\tau_{01} \leqslant \tau \leqslant \tau_{1}$. Let at some instant $\tau_{02}\left(\tau_{02}>\tau_{01}\right)$, the tensile temperature stresses $\sigma^{+}$on the surface $\gamma=h$ reaches the permissible level $\sigma_{*}^{+}$, and when $\tau>\tau_{02}$ they exceed it. With this, the temperature $t^{+}$on the surface $\gamma=h$ of the plate satisfies the condition $t^{+}\left(\tau_{02}\right)<t_{0}$. Here we assume that the tensile stresses $\sigma^{+}$in the time zone $0 \leqslant \tau \leqslant \tau_{01}$ do not exceed the permissible $\sigma_{*}^{+}$. And from the analysis of the Euler equations (28) we obtain:

$$
\lambda_{1}=0, \quad \lambda_{2}=\sum_{i=0}^{n} \lambda_{i 0} \tau^{i}, \quad \phi_{11}^{+}(\tau)=\frac{\pi}{2}, \quad \phi_{1}^{+}(\tau)<\frac{\pi}{2} .
$$

Then, from the third equation (26) for $\phi_{11}^{+}(\tau)=\frac{\pi}{2}$, we obtain

$$
\frac{d T_{1}}{d \tau}+\frac{3 \sigma_{*}^{+}(1-\nu)}{E \alpha_{t} a^{2} h^{2}}=0
$$

From this dependence, we have

$$
T_{1}=\frac{3 \sigma_{*}^{+}(\nu-1)}{E \alpha_{t} a^{2} h^{2}} \tau+A
$$

where the constant $A$, we determine from the condition of continuity of the temperature field at the time $\tau=\tau_{02}$, i.e.:

$$
T_{1}\left(\tau_{02}-0\right)=T_{1}\left(\tau_{02}+0\right), \quad \dot{T}_{1}\left(\tau_{02}-0\right)=\dot{T}_{1}\left(\tau_{02}+0\right)
$$

Since the second condition (52) is satisfied identically under the condition of equality of stresses $\sigma^{+}\left(\tau_{02}-0\right)=\sigma^{+}\left(\tau_{02}+0\right) \equiv \sigma_{*}^{+}$at the instant $\tau=\tau_{02}$, then the constant $A$ is found from the first condition (52). Then, using (51) we obtain the following expressions for $t^{+}(\tau)$ and $\sigma^{+}(\tau)$ :

$$
t^{+}(\tau)=\frac{\sigma_{*}^{+}(\nu-1)}{E \alpha_{t}}\left(1+\frac{3 \tau}{a^{2} h^{2}}\right)+A, \quad \sigma^{+}(\tau)=\sigma_{*}^{+},
$$

where

$$
A=t_{0}\left[\nu_{1} \frac{3}{2} \tau_{02}^{2}+\nu_{2} \frac{\tau_{02}^{2}}{2}\left(\tau_{02}-1\right)+\nu_{3} \tau_{02}^{3}\left(\frac{\tau_{02}}{4}-\frac{1}{3}\right)+\nu_{4} \tau_{02}+\nu_{5}\right]-\frac{3 \sigma_{*}^{+}(\nu-1)}{E \alpha_{t} a^{2} h^{2}} \tau_{02}
$$

\section{Numerical experiment}

The optimal annealing regime $t^{+}$and the corresponding temperature stresses $\sigma^{+}$are determined for the plate of the thickness $2 h=14 \mathrm{~mm}$, made of glass with the following physical and mechanical characteristics $\alpha_{t}=9.1-9.4 \cdot 10^{-6} 1 /$ degree; $\lambda=3.9 \cdot 10^{-3} \mathrm{cal} /(\mathrm{cm} \cdot \mathrm{s} \cdot$ degree $) ; \rho=2.26 \mathrm{~g} / \mathrm{cm}^{3} ; \nu=0.22$; 
$C=0.19 \mathrm{cal} /(\mathrm{g} \cdot$ degree$) ; E=6.4 \cdot 10^{5} \mathrm{~kg} / \mathrm{cm}^{2}$. Maximal heating temperature (annealing temperature) $t_{0}=510^{\circ} \mathrm{C}$. The temperature dependence of the coefficient of temperature expansion is shown in Fig. 1 [16].

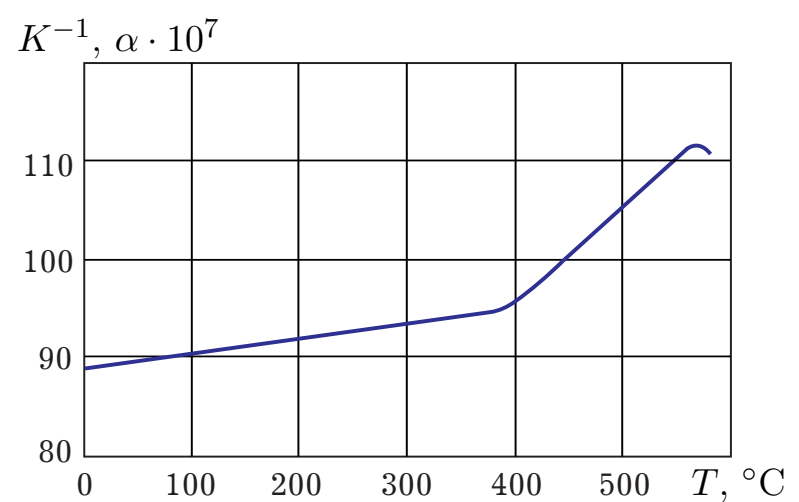

Fig. 1.

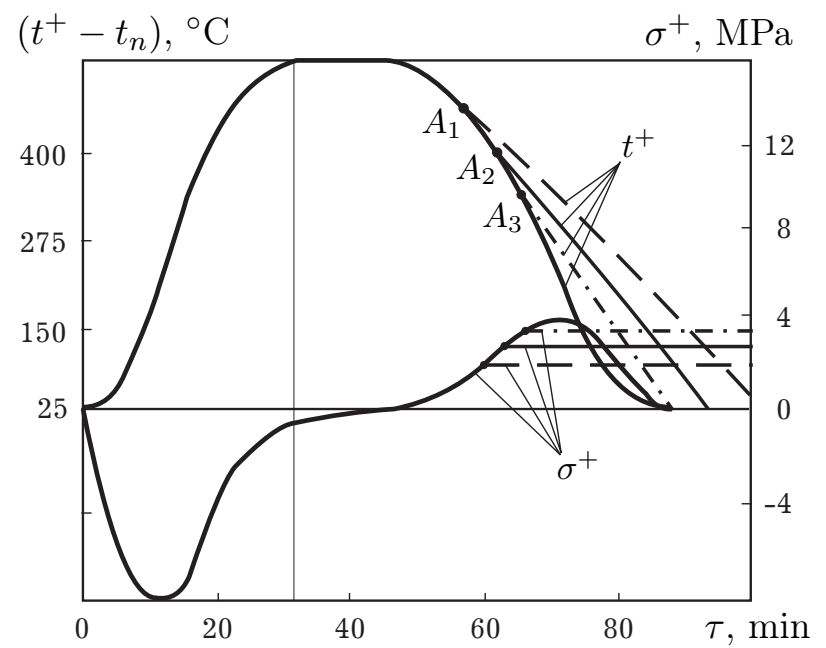

Fig. 2.
In Fig. 2, the changes in time of the optimal annealing regime and the corresponding thermal stresses $\sigma^{+}$for the three values of permissible stresses $\sigma_{*}^{+}$on the surface $\gamma=+h$ of the plate at $\tau_{0}=29 \mathrm{~min}$ and $\tau_{01}-\tau_{0}=10 \mathrm{~min}$ are shown. The dashed curve corresponds to $\sigma_{*}^{+}=2.28 \mathrm{MPa}$, solid curve to $\sigma_{*}^{+}=3.05 \mathrm{MPa}$, dashed dotted curve to $\sigma_{*}^{+}=3.81 \mathrm{MPa}$. It is seen that with the decrease of the value of permissible thermal stress, the duration of annealing increases significantly. With the points $A_{i}, i=\overline{1,3}$, the moments of time $\tau=\tau_{02}$ are indicated at which the stresses $\sigma^{+}$on the surface of the plate reaches the permissible values $\sigma_{*}^{+}$. In this case, the tensile stresses acquire the maximal values at the beginning of cooling (in the vicinity of the time from which cooling begins).

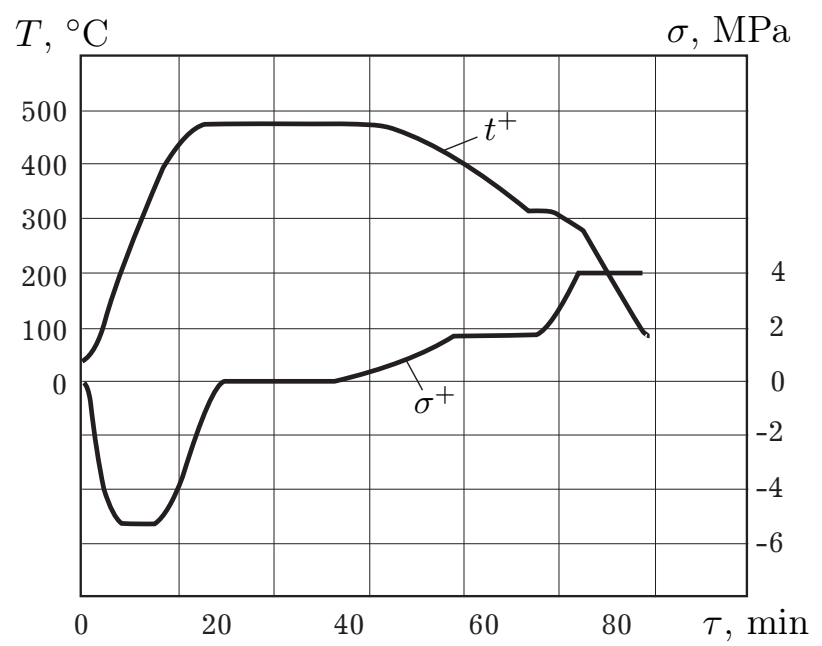

Fig. 3.

The level of these stresses can be reduced in optimal regimes by introducing preliminary auxiliary allowable tensile stresses $\sigma_{* *}^{+} \ll \sigma_{*}^{+}$, at which the temperature gradient and the levels of tensile stresses $\sigma^{+}$are decreasing in the vicinity of the instant of the time from which cooling begins. At the same time, the cooling process becomes two-stage. At the second stage, the cooling process takes place with the true permissible tension $\sigma_{*}^{+}$.

In Fig. 3, the optimal changes of the temperature $t^{+}$in time and of the corresponding stresses $\sigma^{+}$ on the surface $\gamma=+h$ of the plate in the two-stage cooling process are illustrated. The duration of exposure at annealing temperature $\left(\tau_{01}-\tau_{0}\right)=20 \mathrm{~min}$. The proceeding to the maximal temperature is $\tau_{0}=29 \mathrm{~min}$, and the duration of the heating $\tau_{1}=117 \mathrm{~min}$. The solid curves show the optimal changes of surface temperature in time and of the stresses corresponding to it on this surface. It is shown that when heating the plate, from the initial to the annealing temperatures, the temperature stresses are compressive, and when cooling the plate, they are tensile. According to the above-mentioned, the cooling regime is constructed with restrictions on two levels of tensile stresses. At first, when cooling to 
the temperature $330^{\circ} \mathrm{C}$ (under greater of which there are possible structural transformations and the desired minimal temporal tensile stresses), the limitations on tensile temperature stresses are taken of the order of $\sigma_{* *}^{+}=1.7 \mathrm{MPa}$ (small values), then the cooling goes without restrictions until the stresses on the surface of the plates reach the actual permissible $\sigma_{*}^{+}$(of greater values, about $4 \mathrm{MPa}$ ). Cooling with these restrictions continues to the final temperature (output of annealing furnace). With this, the duration of the annealing process increases slightly (see Fig. 2 and Fig. 3). Such a regime at high temperatures of cooling, practically does not lead to the emergence of additional residual deformations.

\section{Conclusions}

The performed numerical studies for the C-93 and C-94 glass (used in electrovacuum production) have shown that the consideration of the thermal sensitivity coefficient of thermal expansion in this case (for the considered plates and shells) results in refining the results within the accuracy of the shell theory (about 5\%). Therefore, when constructing optimal annealing regimes in engineering practice, it is expedient to use the analytical solution of the optimization problem, which is based on minimizing the functional of elastic deformation energy over the time of heating for a direct problem of thermomechanics with constant coefficients of thermal expansion (for the values equal to the mean value on the interval of the temperatures under consideration). Such a solution is used for constructing an initial approximation of the control function.

[1] Grigolyuk E., Podstrigach Ya., Burak Ya. Optimization of heating of shells and plates. Kiev, Naukova dumka (1979), (in Russian).

[2] Biswas P. Thermal Stresses, Deformations and Vibrations of Plates and Shells - A Nonlinear Approach. Procedia Engineering. 144, 1023-1030 (2016).

[3] Podstrigach Ya., Kolyano Yu., Semerak M. Temperature fields and stresses in elements of electrovacuum devices. Kiev, Naukova dumka (1981), (in Russian).

[4] Carrera E., Fazzolari F., Cinefra M. Thermal Stress Analysis of Composite Beams, Plates and Shells. Computational Modelling and Applications. Massachusetts, Academic Press (2016).

[5] Bartenev H. Mechanical properties and heat treatment of glass. Moscow, Stroyizdat (1960), (in Russian).

[6] Bartenev H. High-strength and highly-strength inorganic glasses. Moscow, Stroyizdat (1974), (in Russian).

[7] Pukh V. Strength and destruction of glass. Moscow, Nauka (1973), (in Russian).

[8] Solntsev S., Morozov E. The destruction of glass. Moscow, Mechanical Engineering (1978), (in Russian).

[9] BudzS., Gachkevich N. Optimization of heat treatment of piecewise homogeneous shells of ELB with allowance for the temperature dependence of the material characteristics. Physico-chemical mechanics of materials. 5, 111-113 (1987), (in Russian).

[10] Chernousko F., Banichuk N. Variational problems of mechanics and control. Moscow, Nauka (1973), (in Russian).

[11] Norry D., Freese J. An Introduction to the Finite Element Method. Moscow, Mir (1981).

[12] Gachkevich O., Gachkevich M., Budz S. Optimization under the stress state of the heating modes of glass lump-homogeneous membranes. Lviv, Pidstryhach Institute for Applied Problems of Mech. and Math., National Academy of Sciences of Ukraine (2014), (in Ukrainian).

[13] Korn G., Korn T. Reference on mathematics. Moscow, Nauka (1974).

[14] Marchuk G. Methods of computational mathematics. Moscow, Nauka (1977), (in Russian).

[15] Gachkevich M., Gachkevich O., Torskyy A., Dmytruk V. Mathematical models and methods of optimization of technological heating regimes of the piecewise homogeneous glass shell. State-of-the-art investigations. Mathematical Modeling and Computing. 2 (2), 140-153 (2015).

[16] Baranovsky V., Gusev V., Ivanov V. and others. Production of color kinescopes. Ed. Baranovsky V. Moscow, Energia (1978), (in Russian).

Mathematical Modeling and Computing, Vol.5, No.2, pp. 134-146 (2018) 


\title{
Математична модель оптимізації за напруженим станом режимів відпалу термочутливих скляних елементів конструкцій
}

\author{
Гачкевич O. ${ }^{1,2}$, Гачкевич M. ${ }^{1}$, Торський А. ${ }^{3}$, Дмитрук B. ${ }^{4,3}$ \\ ${ }^{1}$ Інститут прикладних проблем механіки і математики \\ ім. Я. С. Підстригача НАН України, вул. Наукова, 3-б, Лъвів, 79060, Україна \\ ${ }^{2}$ Політехніка Опольска, вул. Прушковсъка, 76, Ополе, 45-758, Польща \\ ${ }^{3}$ Центр математичного моделювання Інституту прикладних проблем механіки $i$ математики \\ ім. Я. С. Підстригача НАН України, вул. Д. Дудаєва, 15, 79005, Лъвів, Украӥна \\ ${ }^{4}$ Національний університет “Лъвівсъка політехніка", вул. С. Бандери, 12, Лъвів, 79013, Україна
}

Побудовано математичну модель і розв'язано задачу оптимізації режимів відпалу термочутливих скляних елементів конструкцій з використанням числових методів і варіаційного числення. Як приклад побудовано і проаналізовані оптимальні режими відпалу конкретних скляних пластин за різних значень допустимих напружень.

Ключові слова: теплові режими в виробничих процесах, оптимізація відносно напруженого стану, термочутливі скляні елементи.

2000 MSC: 74A10, 74B10

УдК: 539.3 